\title{
Instilled Epinephrine into the Oropharynx for Life Threatening Post-Tonsillectomy Hemorrhage
}

\author{
Donna Mendez* \\ Department of Emergency Medicine, USA
}

Received: 畊 January 17, 2019; Published: 制January 28, 2019

*Corresponding author: Donna Mendez, Department of Emergency Medicine, USA

\begin{abstract}
Post-Tonsillectomy Hemorrhage (PTH) is one of the more lethal complications after a tonsillectomy and if one survives there is significant neurologic morbidity due to hypoxia. A 7-year-old female patient with PTH presented to an Emergency Department requiring intubation and cardiac resuscitation. In this case the use of instilled liquid epinephrine administered down the oropharynx allowed intubation and full recovery of the patient.
\end{abstract}

Abbreviations: PTH: Post Tonsillectomy Hemorrhage; LMA: Laryngeal Mask Airway; DL: Direct Laryngoscopy; FI: Flexible Scope Intubation; ED: Emergency Department; IV: Intravenous Line

\section{Introduction}

Post-Tonsillectomy Hemorrhage (PTH) is one of the more lethal complications after a tonsillectomy. It occurs in up to $3 \%$ of all patients [1]. The mortality is low, reported as $<1 \%[2,3]$ however, morbidity has been reported as $20 \%$ [4]. PTH is classified as primary or secondary hemorrhage. Primary hemorrhage after a tonsillectomy is bleeding that occurs in the first 24 hours and is attributed to the surgical procedure itself. A secondary hemorrhage is bleeding that starts more than 24 hours after tonsillectomy and is associated with clot sloughing from the tonsillar fossa and often unrelated to surgical technique. Secondary bleeding occurs an average of 7 days after tonsillectomy and is far more common than primary bleeding $[5,6]$.

There is little evidence available on the management of PTH. Among recent studies, some investigators have suggested admission to the hospital of all patients with a history of PTH and recommended electrocautery or suture ligature in the operating room for any clot or active bleeding noted [7,8]. Others have described several methods of initial management that range from observation to bedside intervention with silver nitrate, topical or injected vasoconstrictors or bipolar cautery [8-13]. In a survey by the American Society of Pediatric Otolaryngologists in 2017, the majority $(82 \%)$ of otolaryngologists agreed that control of the hemorrhage should occur in the operating room after topical treatment [14]. Most otolaryngologists agree that for massive hemorrhage, ligation of the ipsilateral external carotid artery is recommended in the operating room [15]. Since intubation is difficult due to arterial bleeding in massive PTH, failed intubation is a possibility. Recommendations are that immediate surgical airway such as a cricothyrotomy should be performed if one cannot intubate by traditional direct laryngoscopy (DL) or alternative airways such as laryngeal mask airway (LMA), flexible scope intubation (FI) or video laryngoscopy (VL) [16].

Some otolaryngologists have recommended limited intubation attempts to a maximum of two, or within two minutes, to allow enough time for cricothyrotomy to avoid severe hypoxia [2]. An immediate airway is necessary since children have a lower capacity for residual pulmonary function and consume more oxygen per hour, so even a short time of apnea could result in hypoxia. However, a surgical airway is not typically used in children aged eight years or younger in contrast to adults and needle cricothyrotomy recommended in children younger than eight years of age since their airway structures are smaller [17-19].

\section{Case Report}

A $7 y / 0$ female was brought into the emergency department (ED) by ambulance for decreased level of consciousness. She had no fever or other preceding symptoms. Per the paramedics she fainted at home, and when she regained consciousness, she was drowsy and pale, but her GCS was 10 and vital signs normal, so she was not intubated and transported to the ED. Paramedics inserted an intravenous line (IV) prior to arrival. When she presented to the ED, she was very pale and lethargic. Her vital signs included 
an HR of 170 BP of 90/50 RR 20 oxygen saturation of $100 \%$ on $100 \%$ oxygen by nonrebreather with her GCS being 6 . Due to her level of consciousness, she was prepared for intubation with rapid sequence intubation. The resident attempted intubation which was unsuccessful on the first attempt. The attending then attempted and was unsuccessful after the first attempt and then after the laryngoscope was withdrawn from the oropharynx, blood filled the oropharynx immediately. Suctioning was performed but did not clear the airway even to visualize the oropharynx. A Laryngeal Mask Airway (LMA) was inserted but clotted off with blood, and we were unable to oxygenate or ventilate the patient. The LMA was pulled out and then the patient became bradycardic with an HR of 55 so CPR was started and epinephrine $.01 \mathrm{mg} / \mathrm{kg}$ of 1:1,000 given through an IV with an improvement of the HR to 100 . The patient was quickly transfused with 0 negative blood since she had lost approximately 1.5 liters of blood in a matter of 10 minutes. In an attempt to clot off the blood from the oropharynx so an airway could be obtained, epinephrine. $1 \mathrm{mg} / \mathrm{kg}$ of 1:1,000 followed by 5 ccs of normal saline was then instilled down the oropharynx. Approximately 1 minute after epinephrine was administered the bleeding stopped and the patient was successfully intubated. The patient was taken directly to the operating room where there was an arterial injury identified requiring ligation of the ipsilateral external carotid artery. Injury to the artery was felt to be due to accidental injury during the tonsillectomy. She received another blood transfusion in the OR and was found to have no underlying bleeding disorder. The patient was extubated after the surgery and was discharged home in two days without any sequela.

\section{Discussion}

Post-Tonsillar hemorrhage (PTH) is a relatively common condition after tonsillectomy. It is rarely life-threatening as in our patient. The reports of morbidity and mortality have been due to not obtaining an airway due to copious amounts of blood in the oropharynx. Those in which a cricothyrotomy was performed did not survive or had neurological sequela [4]. Unfortunately, there is little evidence on the management of massive PTH without a standardized guideline published. Since it is imperative to obtain an airway quickly most otolaryngologists agree that if standard and alternative airway interventions are unsuccessful a cricothyrotomy is recommended. In our case, DL or LMA was not possible due to massive bleeding however there is one report of LMA being successfully placed in a 6-year-old child with massive PTH has been published [20].

The treatment of PTH with instilled epinephrine down the oropharynx has not been reported in the literature. There have been limited reports of topical vasoconstrictors, such as epinephrine, for initial management. There is a report of aerosolized epinephrine for mild hemorrhage due to spontaneous tonsillar bleed [21]. There are reports of gauze or cotton pack soaked with epinephrine, epinephrine -lidocaine combination or cocaine to stop PTH $[22,23]$. More recent reports recommend soaked epinephrine gauze be applied directly to the tonsillar fossa with McGill forceps for nonmassive PTH. The only literature for airway hemorrhage with instilled liquid epinephrine has been for airway bleeding due to bronchoscopy. One of the risks with instilled liquid down the airway is cardiac dysrhythmias, hypotension, and coronary spasm [24,25]. The Cleveland Clinic practice over the past 32 years has used 2-ml aliquots of 1:10:000 epinephrine up to a maximum dose of $0.6 \mathrm{mg}$ without any adverse side effects [26].

We would recommend a dose of epinephrine 1:100,000 in small ( $2 \mathrm{ml}$ ) aliquots up to a maximum dose of $0.6 \mathrm{mg}$ in a patient who is not in a cardiac arrest situation. Our patient was given a dose of .1mg/kg of 1:1,000 down the airway which is four times the recommended .6mg maximum dose but a lower dose may have been sufficient to stop the hemorrhage.

\section{Conclusion}

Our case was a demonstration of a lifesaving procedure in a child with a difficult airway due to massive PTH. It is critical that an emergency department physician can manage massive post tonsillar hemorrhage since this is where patients routinely present. The use of epinephrine instilled down the airway when an airway cannot be obtained due to hemorrhage with DL, VL or LMA may be tried prior to attempting a surgical airway.

\section{References}

1. De Luca Canto G, Pacheco Pereira C, Aydinoz S, Bhattacharjee R, Tan HL, et al. (2015) Adenotonsillectomy Complications: A Meta-analysis. Pediatrics 136(4): 702-718.

2. Cohen D, Dob M (2008) Morbidity and mortality of post-tonsillectomy bleeding: analyses of cases. J Laryngol Oto 122(1): 88-92.

3. Østvoll E, Sunnergren O, Ericsson E, Hemlin C, Hultcrantz E, et al. (2015) Mortality after tonsil surgery, a population study, covering eight years and 82,527 operations in Sweden. Eur Arch Otorhinolaryngol 272(3): 737-743.

4. Windfuhr JP, Schloendorff G, Baburi D, Kremer B (2008) Serious posttonsillectomy hemorrhage with and without lethal outcome in children and adolescents. Int J Pediatr Otorhinolaryngol 72(7):1029-1040.

5. Steketee KG, Reisdorf EJ (1995) Emergency care for post-tonsillectomy and postadenoidectomy hemorrhage. Am J Emerg Med 13(5): 518-523.

6. Chowdhury K, Tewfik TL, Schloss MD (1988) Post-tonsillectomy and adenoidectomy hemorrhage. J Otolaryngol 17(1): 46-49.

7. Miller L Miller, KH Richmond L (1986) Post-tonsillectomy hemorrhage: incidence, prevention and management. Laryngoscope 96(11): 12431247.

8. Peterson J, Losek JD (2004) Post-tonsillectomy hemorrhage and pediatric emergency care. Clin Pediatr 3(5): 445-448.

9. Wei JI, Beatty CW, Gustafson RO (2000) Evaluation of posttonsilectomy hemorrhage and risk factors Otolaryngol Head Neck Surg 23(3): 229235.

10. Liu JH, Anderson KE, Willging JP, Myer CM, Shott SR, et al. (2001) Post tonsillectomy hemorrhage: what is it and what should be recorded? Arch Otolaryngol Head Neck Surg 127(10): 1271-1275.

11. Kim DW, Koo JW, Ahn SH, Lee CH, Kim JW, et al. (2010) Difference of delayed post-tonsillectomy bleeding between children and adults. Auris Nasus Larynx 37(4): 456-460.

12. Attner P, Haraldsson PO, Hemlin C, Hessén Soderman AC (2009) A 4-year consecutive study of post-tonsillectomy haemorrhage, ORL J Otorhinolaryngol Relat Spec 71(5): 273-238. 
13. Irani DB, Berkowitz RG (1997) Management of secondary hemorrhage following pediatric adenotonsillecttomy. Int Pediatr Otorhinolaryngol 40(2-3): 115-124.

14. Clark CM, Schubart JR, Carr MM (2018) Trends in the management of secondary post-tonsillectomy hemorrhage in children. Int J Pediatr Otorhinolaryngo 108:196-210.

15. Handler SD, Miller L, Richmond KH, Baranak CC (1966) Posttonsillectomy hemorrhage: incidence, prevention and management Laryngosope 96(11): 1243-1247.

16. Jagannathan N, Burjec N (2019) Management of the difficult airway for pediatric anesthesia. Hagberg CA, Sun LS, Crowley M (Eds.), UptoDate.

17. Coté CJ, Hartnick CJ (2009) Pediatric transtracheal and cricothyrotomy airway devices for emergency use: which are appropriate for infants and children? Paediatr Anaesth 19(1): 66-76.

18. Goumas P, Kokkinis K, Petrocheilos J, Naxakis S, Mochloulis G, et al. (1997) Cricothyrotomy and the anatomy of the cricothyroid space. An autopsy study. J Laryngol Otol 111(4): 354-356.

19. Navsa N, Tossel G, Boon JM (2005) Dimensions of the neonatal cricothyroid membrane-how feasible is a surgical cricothyroidotomy? Paediatr Anaesth 15(5): 402-426.

\section{ISSN: 2574-1241}

DOI: 10.26717/BJSTR.2019.13.002438

Donna Mendez. Biomed J Sci \& Tech Res

(c) This work is licensed under Creative

Submission Link: https://biomedres.us/submit-manuscript.php
20. Go WH, Kim KT, Kim JY, Won Joo Choe, Jung Won Kim, et al. (2012) The use of laryngeal mask airway in pediatric patient with massive posttonsillectomy hemorrhage. Korean J Anesthesiol 63(2): 177-178.

21. Rowlands RG, Hicklin L, Hinton AL (2002) Novel use of nebulized adrenaline in the treatment of secondary oropharyngeal haemorrhage. J Laryngol Oto 116(4): 123-124.

22. Steketee KG, Reisdorff J (1995) Emergency Care for Posttonsillectomy and Postadenoidectomy Hemorrhage. Am J Emerg Med 13(5): 518-523.

23. Fox SM (2012) Post-Tonsillectomy Hemorrhage. Pediatric Morsels.

24. Mall W, Abel H (1978) Topical application of epinephrine during bronchoscopy in barbiturate-halothane anesthesia and its influence on cardiac action. Bronchopneumologie 28(4): 311-316.

25. Vaknin Z, Manisterski Y, Ben Abraham R, Efrati O, Lotan D, et al. (2001) Is endotracheal adrenaline deleterious because of the beta-adrenergic effect? Anesth Analg 92(6): 1408-1412.

26. Khoo KL, Lee P, Mehta AC (2013) Endobronchial epinephrine: confusion is in the air. Am J Respir Crit Care Med 187(10): 1137-1138.

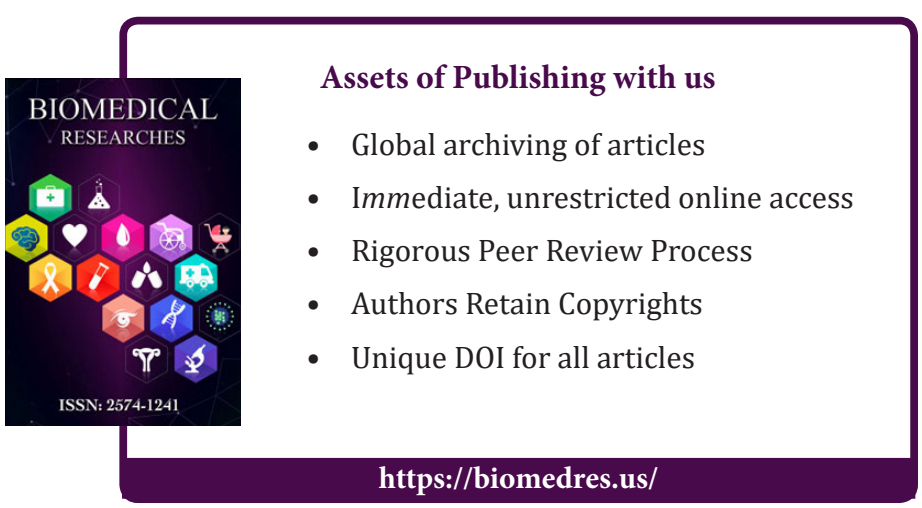

